\title{
Analog Circuit Fault Diagnosis Method Based on Preferred Wavelet Packet and ELM
}

\author{
Haitao Shi ${ }^{1}$, Qide Tan ${ }^{1, *}$, Chenggang $\mathrm{Li}^{2}$ and Xiangyu $\mathrm{Lv}^{2}$ \\ ${ }^{1}$ Northeast Electric Power University, Jilin 132012, China \\ ${ }^{2}$ Electric Power Research Institute, Jilin Electric Power Company Limited, Changchun 130021, China \\ ${ }^{*}$ Corresponding author
}

\begin{abstract}
In order to improve the effectiveness of fault feature extraction and achieve the accurate classification of fault patterns in analog circuit, the paper proposed a new analog circuit fault diagnosis method based on preferred wavelet packet and extreme learning machine (ELM). The concept of feature departure degree is defined, which can be used as a measure of wavelet packet transform to obtain the fault features using different wavelet basis function, and the wavelet basis function with maximum feature departure degree is selected and used to extract the fault feature. Further, the ELM is introduced for fault classification and identification, and the diagnosis result is compared with those using three popular neural networks. The simulation results show that the better diagnosis precision can be achieved using the preferred wavelet packet, and the test time and the classification precision of the ELM are all better than those using other methods.
\end{abstract}

Keywords-wavelet packet transform; extreme learning machine (ELM); analog circuit; fault diagnosis; feature departure degree

\section{INTRODUCTION}

Fault diagnosis technology is a key technology to ensure the normal operation of complex electronic systems, and it has been a hot topic that is concerned by many researchers around the world. Some researches have shown that the analog circuit is the most prone to failure in complex electronic systems. Because of the non-linearity and tolerance limit, traditional fault diagnosis methods, such as fault dictionary method, component parameter identification method and fault verification method, are not applicable.

Neural network is widely used in the field of analog circuit fault diagnosis due to the characteristics of distributed parallel processing, nonlinear mapping, adaptive learning and robust fault tolerance ${ }^{[2-5]}$.Because of its good time-frequency characteristics, wavelet transform has become the most widely used method for fault feature extraction of analog circuits. However, at present, the wavelet basis function selection is more random, and can not guarantee the wavelet decomposition to get the best fault feature. The support vector machine based on statistical learning theory has improved its generalization performance compared with traditional neural networks. However, due to the need to specify the kernel function and kernel parameters, the application of SVM is seriously restricted ${ }^{[11-12]}$.

Because the support vector machine does not need to adjust the hidden layer parameters repeatedly, and the traditional single hidden layer feed-forward neural network parameter training problem is transformed into solving the linear equations. The process is completed at one time. In this paper, an optimalwavelet basis function is proposed for fault feature extraction and combined with an extreme learning machine for analog circuit fault diagnosis.

\section{THE PRINCIPLE OF ELM}

The principle of ELM is different from the traditional feed-forward neural network method which needs to adjust all the parameters to minimize the cost function. The thehods of ELM indicate that the hidden layer learning parameters can be generated randomly. And the output weights can be obtained by solving linear equations. This makes the training speed of extreme learning machine greatly improved, and can achieve better generalization performance.

Given $\mathrm{N}$ training samples $\left\{\left(x_{i}, t_{i}\right)\right\}_{i=1}^{N}$, the input sample $x_{i}=\left[x_{i 1}, x_{i 2}, \cdots, x_{i n}\right]^{T} \in R^{n}$, the output sample $t_{i}=\left[t_{i 1}, t_{i 2}, \cdots, t_{i n}\right]^{T} \in R^{m}$, the output of SLFNs with $\tilde{N}$ hidden nodes and activation function $\mathrm{G}$ (wj, bj, xi) is:

$$
o_{i}=\sum_{j=1}^{N} \beta_{j} G\left(w_{j}, b_{j}, x_{i}\right) ; i=1, \cdots, N
$$

where: $w_{j}=\left[w_{j 1}, w_{j 2}, \cdots, w_{j n}\right]^{T}$ is the weight vector connecting the $\mathrm{jth}$ hidden layer neuron and the input neuron; $\beta_{i}=\left[\beta_{i 1}, \beta_{i 2}, \cdots, \beta_{i n}\right]^{T}$ is the weight vector connecting the jth hidden layer neuron and the output neuron; $b_{j}$ is the deviation of the jth hidden layer neuron.

In order that SLFNs is zero error approximation of the $\mathrm{N}$ samples, the following formula holds:

$$
\sum_{j=1}^{\widetilde{N}} \beta_{j} G\left(w_{j}, b_{j}, x_{i}\right)=t_{i} ; i=1, \cdots, N
$$

The $\mathrm{N}$ equations of formula (2) can be abbreviated as:

$$
H \beta=T
$$


where: $\mathrm{H}$ is called the hidden layer output matrix of the neural network. In most cases, the number of hidden layer nodes is far less than the number of training samples. In this time, wi, $\beta_{i}$, bi, $(\mathrm{i}=1, \ldots, \tilde{N})$ can not Satisfy the formula $H \beta=T$. According to the generalized inverse lemma, the least-squares solution of the above linear system is:

$$
\hat{\beta}=H^{+} T
$$

In summary, a training sample set, the activation function $\mathrm{G}(\mathrm{x})$ and the number of hidden nodes is given, the step of ELM learning algorithm is:

1) The input weight wi and the hidden layer deviation $b_{i}$ are specified at random.

2) To calculate the hidden layer output matrix $H$.

3) To calculate the output weight value $\beta$.

For multiple classification problems, ELM is implemented by multiple output regression algorithm.

\section{OPTIMAL FAULt FEATURE EXTRACTION}

\section{A. Fault Feature Extraction Based on Wavelet Packet Transform}

Wavelet packet analysis is a generalization of multiresolution analysis. It divides the frequency band into several layers, and inherits the advantage of time-frequency localization of wavelet transform. At the same time, it decomposes the high-frequency part which is not subdivided by multi-resolution analysis, and has better time-frequency characteristics.

Because the wavelet transform only decomposes the low-frequency part of the signal and the detail part of the signal does not continue to decompose. Therefore, the wavelet transform can represent a large class of low frequency information as the main component of the signal. But it does not decompose the signal well and represent the signal that contains the detail information. The wavelet packet transform can provide finer resolution for the high frequency part, and this decomposition has no redundancy and omission. It can analyze the frequency and time resolution of the high frequency and low frequency band of the signal at the same time.

The wavelet packet function is defined as:

$$
u_{j, k}^{n}=2^{j / 2} \mu^{n}\left(2^{j} t-k\right)
$$

where: $\mathrm{n}$ is the oscillation parameters; $\mathrm{J}$ and $\mathrm{k}$ are scale parameters and translation parameters, respectively.

When $\mathrm{n}=0,1 ; \mathrm{j}=\mathrm{k}=0$, the initial two wavelet packet functions are defined as:

$$
\mu_{0}(t)=\varphi(t), \mu_{1}(t)=\psi(t)
$$

The initial wavelet packet function satisfies the two-scale equation:

$$
\left\{\begin{array}{l}
\mu_{0}(t)=\sqrt{2} \sum_{k \in Z} h(k) \mu_{0}(2 t-k) \\
\mu_{1}(t)=\sqrt{2} \sum_{k \in Z} g(k) \mu_{0}(2 t-k)
\end{array}\right.
$$

where: $\mathrm{h}(\mathrm{k})$ and $\mathrm{g}(\mathrm{k})$ are the corresponding multi-scale analysis of low-pass filter coefficients and high-pass filter coefficients. Then when $n=2,3, \ldots$, the other wavelet packet functions satisfy the formula:

$$
\left\{\begin{array}{l}
\mu_{2 n}(t)=\sqrt{2} \sum_{k \in Z} h(k) \mu_{n}(2 t-k) \\
\mu_{2 n+1}(t)=\sqrt{2} \sum_{k \in Z} g(k) \mu_{n}(2 t-k)
\end{array}\right.
$$

For a set of discrete signals $\mathrm{x}(\mathrm{t})$, the wavelet packet decomposition and reconstruction algorithms are as follows:

$$
\begin{aligned}
& \left\{\begin{array}{l}
d_{j+1}^{2 n}=\sum_{k} h(k-2 t) d_{j}^{n}(k) \\
d_{j+1}^{2 n}=\sum_{k} g(k-2 t) d_{j}^{n}(k)
\end{array}\right. \\
& d_{j}^{n}(k)=2\left[\sum_{\tau} h(k-2 \tau) d_{j+1}^{2 n+1}(k)+\right. \\
& \left.\sum_{\tau} g(k-2 \tau) d_{j+1}^{2 n}(k)\right]
\end{aligned}
$$

$d_{j}^{n}(k)$ denotes the $\mathrm{k}$-th coefficient corresponding to the node $(j, n)$ after the wavelet packet decomposition, and the node $(j, n)$ denotes the $n$-th frequency band of the $\mathrm{j}$-th layer.

The wavelet packet process is used to extract the fault features of analog circuits. The output signals are decomposed by wavelet packet in the normal state and fault state of the analog circuits. Then the normalized energy of each frequency band is used as the characteristic input for training and testing.

\section{B. Preferably Wavelet Packet Decomposition}

When decomposing the circuit response using wavelet, the different wavelet basis functions will obtain different results. In order to extract the effective features of the circuit, we must choose the optimal wavelet basis for wavelet packet decomposition. Therefore, this paper proposes the concept of feature deviation as the selection measure to determine the optimal wavelet base, which is defined as: the deviation between the fault feature and the normal state feature can be calculated by formula (11).

$$
D_{b}=\sum_{i=1}^{s} \sum_{j=1}^{N} P_{i}\left(m_{j}^{i}-m_{j}\right)\left(m_{j}^{i}-m_{j}\right)^{T}
$$

where: $\mathrm{Pi}$ is the prior probability of the i-th fault feature; 
$m_{i}^{j}$ is the $\mathrm{j}$-th data in the $\mathrm{i}$-th set of fault feature samples; $\mathrm{m}_{\mathrm{j}}$ is the $j$-th data in the normal fault sample set. If the characteristic deviation degree is bigger, it indicates that the fault feature is more effective. And it is more conducive to isolate different types of fault.The steps of adaptively determining the wavelet basis function are:

1) The output response of the circuit is decomposed by different wavelet bases, and the characteristic deviation is calculated by the obtained fault feature. The wavelet base corresponding to the maximum $\mathrm{Db}$ is the optimal wavelet base.

2) The optimal wavelet basis is selected to decompose the output response of each state to obtain the optimal fault feature.

\section{WaVElet Packet ANd Elm Fault Diagnosis}

As a single-layer feed-forward neural network with excellent performance, especially fewer parameters to be determined and less learning time, it is expected to be better than the present method in the field of analog circuit fault diagnosis. In this paper, the wavelet packet decomposition is used to extract the fault characteristic vector of the analog circuit output response, and then input to ELM for training. Finally, we use the trained extreme learning machine to determine which type of fault the new sample belongs to. The flowchart is shown in Figure I.

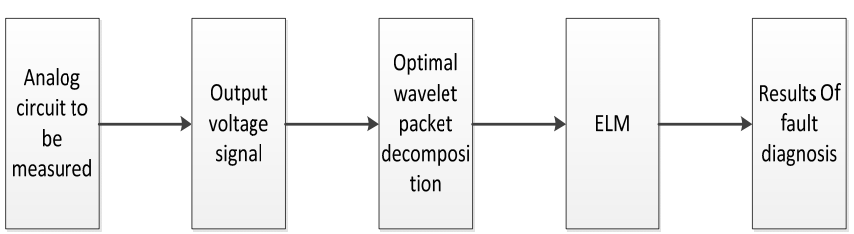

FIGURE I. DIAGNOS IS FLOW CHART OF WAVELET ELM

The specific steps are as follows:

(1) To analyze the sensitivity of the measurement circuit and selecte the components which have great influence on the output as the fault components, then extract the output response.

(2) The optimal wavelet basis function is used to decompose the output response signal by 3 layer wavelet packet decomposition, and the fault characteristic samples are obtained.

(3) The fault feature samples are input to ELM for training.

(4) The new fault feature samples are input into the trained ELM for fault discrimination.

\section{V.ANALYSIS OF THE EXAMPLES}

Selecting the band pass filter circuit showen in Figure II to conduct experiment, and the tolerance of the resistance and and capacitance were $5 \%$ and $10 \%$ respectively. This paper only studies the single circuit failure for resistance and capacitance.

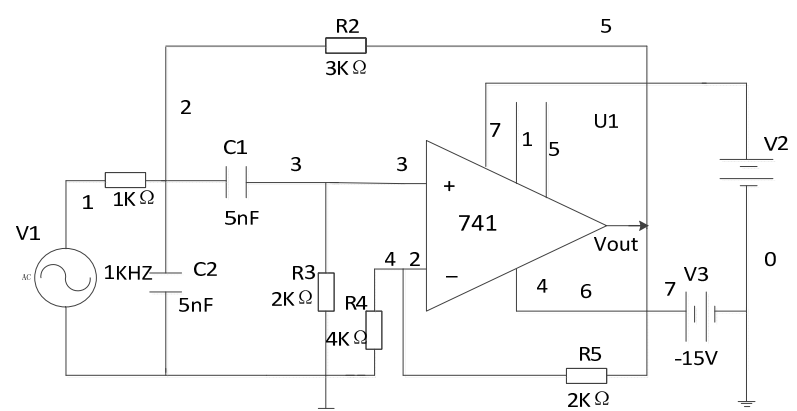

FIGURE II. THE BAND-PASS FILTER CIRCUIT USED IN DIAGNOSIS

The sensitivity analysis of the circuit using Multisim circuit simulation software shows that the change of R2, R3, $\mathrm{C} 1$ and $\mathrm{C} 2$ has a great influence on the circuit output, so choose the four components for diagnostic analysis. Setting the value range of fault component is $[50 \% \mu,(1-t) \mu]$ $\mathrm{U}[(1+t) \mu, 150 \% \mu]$, where $\mathrm{t}$ is the component tolerance and $\mathrm{u}$ is the component nominal value. A total of nine fault modes including the normal state can be obtained, and the nominal value and fault value range are shown in Table 1.

TABLE I. FAULT MODES

\begin{tabular}{|l|l|l|}
\hline Fault Modes & Nominal Value & Fault value range \\
\hline $\mathrm{C}_{1} \uparrow$ & $5 \mathrm{nF}$ & {$[5.5 \mathrm{nF}, 7.5 \mathrm{nF}]$} \\
\hline $\mathrm{C}_{1} \downarrow$ & $5 \mathrm{nF}$ & {$[2.5 \mathrm{nF}, 4.5 \mathrm{nF}]$} \\
\hline $\mathrm{C}_{2} \uparrow$ & $5 \mathrm{nF}$ & {$[5.5 \mathrm{nF}, 7.5 \mathrm{nF}]$} \\
\hline $\mathrm{C}_{2} \downarrow$ & $5 \mathrm{nF}$ & {$[2.5 \mathrm{nF}, 4.5 \mathrm{nF}]$} \\
\hline $\mathrm{R}_{2} \uparrow$ & $3 \mathrm{~K} \Omega$ & {$[3.15 \mathrm{~K} \Omega, 4.5 \mathrm{~K} \Omega]$} \\
\hline $\mathrm{R}_{2} \downarrow$ & $3 \mathrm{~K} \Omega$ & {$[1.5 \mathrm{nF}, 2.75 \mathrm{nF}]$} \\
\hline $\mathrm{R}_{3} \uparrow$ & $2 \mathrm{~K} \Omega$ & {$[2.1 \mathrm{~K} \Omega, 3 \mathrm{~K} \Omega]$} \\
\hline $\mathrm{R}_{3} \downarrow$ & $2 \mathrm{~K} \Omega$ & {$[1 \mathrm{~K} \Omega, 1.9 \mathrm{~K} \Omega]$} \\
\hline
\end{tabular}

The simulation of the 9 failure modes is carried out, and selecting the $\mathrm{db} 3$ wavelet decomposes the output response of each state to obtain the optimal fault feature set. In order to compare with the wavelet extreme learning machine, BPNN, RBFNN and LS-SVM are separately selected to diagnose the tested circuit respectively.

TABLE II. DIAGNOSIS RESULTS FOR FOUR METHODS

\begin{tabular}{|l|l|l|l|}
\hline Method Type & Training Time & Testing Time & $\begin{array}{l}\text { Diagnostic } \\
\text { Accuracy } \\
(\%)\end{array}$ \\
\hline BP & 6.24580 & 0.01300 & 85.81 \\
\hline RBF & 0.04060 & 0.00890 & 86.67 \\
\hline LS-SVM & 0.05460 & 0.04990 & 91.11 \\
\hline ELM & 0.00154 & 0.00079 & 94.44 \\
\hline
\end{tabular}

From the diagnosis results, ELM diagnosis accuracy is significantly higher than the other three methods, and training 
time and testing time are significantly less than the other three methods. The use of ELM analog circuit fault diagnosis can attain the most accurate results.

\section{CONCLUSION}

In order to extract the optimal fault feature by wavelet packet transform, the concept of characteristic deviation is put forward. A diagnosis method of optimal wavelet packet and extreme learning machine is proposed to overcome the shortcomings of traditional artificial intelligence method in fault diagnosis of analog circuit. This method combines the advantage of wavelet transform and extreme learning machine, and realizes the fast and accurate diagnosis of analog circuit fault. The simulation results of bandpass filter show that the diagnostic accuracy of the proposed wavelet extreme learning machine can reach to $94.44 \%$, so the performance far exceeds the other three commonly used fault diagnosis methods.

\section{ACKNOWLEDGMENT}

In writing this paper, I have benefited from the presence of my teachers and my classmates. They generously helped me collect materials I needed and made many invaluable suggestions. I hereby extend my grateful thanks to them for their kind help. My parents took good care of my life and gave me a lot of encouragement when I was working at this thesis. They always share my weal and woe. I feel much grateful and heartily owe my achievement to them.

\section{REFERENCES}

[1] YANG X, FANG H J. Summary of neural network approach to analog circuit fault diagnosis [J]. Computer Measurement \& Control, 2006 , 14(5): 564- 566

[2] SONG G M, WANG H J, JIANG SH Y, et al. Analog circuit fault diagnosis approach using clustering and hierarchical decision SVM[J]. Chinese Journal of Scientific Instrument, 2010, 31(5): 998- 1004

[3] MA CH, CHEN X H, XU Y L., et al. Analog circuit fault diagnosis based on attribute reduct ensemble of support vector machine[J]. Chinese Journal of Scientific Instrument, 2011, 32 (3): 660- 666.

[4] LUO H, WANG Y R, CUI J. A SVDD approach of fuzzy classification for analog circuit fault diagnosis with FWT as preprocessor [J]. Expert Systems with Applications, 2011, 38: 10554- 10561

[5] CUI J, WANG Y R. A novel approach of analog circuit fault diagnosis using support vector machines classifier [J]. Measurement, 2011, 44: 281- 289 .

[6] AMINIAN M, INIAN F. Neural-network based analog-circuit fault diagnosis using wavelet transform as preprocessor [J]. IEEE Trans. on Circuits and Systems, 2000, 47(2): 151- 156.

[7] YUAN H Y, SUN X, LI H T. Fault feature recognition in analog circuit based on lifting wavelet $[\mathrm{J}]$. Journal of Electronic Measurement and Instrument, 2011, 25 (8): 700- 703.

[8] TIAN CH L, PENG M F, SONG L W, et al. Optimal fault feature search and recognition in analog circuit $[\mathrm{J}]$. Chinese Journal of Scientific Instrument, 2012, 33 (7):1549- 1555.

[9] TAN Y H, HE Y G. A novel method for fault diagnosis of analog circuits based on WP and GPNN [J]. International Journal of Electronics, 2008, 95(5): 431-439.

[10] ZHU W J, HE Y G. A GA-BPNNs based approach for fault diagnosis of analog circuits and its application [J]. Journal of Computer-aided Design \& Computer Graphics, 2009, 21(9): 1283- 1289.

[11] SUN J, WANG CH H. Analog circuit fault diagnosis based on mRMR and optimized SVM [J]. Chinese Journal of Scientific Instrument, 2013, 34(1): 221- 226.

[12] CHEN SH J, LIAN K, WANG H J. Method for analog circuit fault diagnosis based on GA optimized SVM [J]. Journal of University of Electronic Science and Technology of China, 2009, 38(4) : 553- 558

[13] HUANG G B, ZHU Q Y, SIEW C K. Extreme learning machine: Theory and applications [J]. Neurocomputing, 2006, 70: 489- 501.

[14] HUANG G B, XIAO J D, ZHOU H M. Optimization method based extreme learning machine for classification [J]. Neurocomputing, 2010 74: $155-163$.

[15] CAO J, LIN Z, HUANG G B, et al. Voting based extreme learning machine [J]. Information Sciences, 2012. 185: 66- 77.

[16] BAUMANN R. Soft errors in advanced computer systems[J]. IEEE Transactions on Design \& Test of Computers, 2005, 22 (3): 258- 266.

[17] ALIPPI C, CATELANI M, FORT A, et al. Automated selection of test frequencies for fault diagnosis in analog electronic circuits IEEE [J]. Transactions on Instrumentation and Measurement, 2005, 54(3): 10331044.

[18] AMINIAN M, AMINIAN F. A modular fault diagnostic system for analog electronic circuits using neural networks with wavelet transform as a preprocessor [J]. IEEE transactions on Instrumentation and Measurement, 2007, 56(5): 1546- 1554.

[19] HUANG C L, WANG C J. A GA-based feature selection and parameters optimization for support vector machines. Expert Systems with Applications, 2006, 31(2): 231- 240.

[20] YUAN H, CHEN G, XIE Y. Feature extraction based on neural network in analog circuit fault diagnosis [J]. Journal of Systems Engineering and Electronics, 2007, 18 (2): 434- 437.

[21] DING C, PENG H. Minimum redundancy feature selection from microarray gene expression data $[\mathrm{J}]$. Journal of Bioinformatics and Computational Biology, 2005, 3(2): 185- 205. 